\title{
On the short-term retention of serial, tactile stimuli*
}

\author{
EDITH V. SULLIVAN and M. T. TURVEY $\dagger$ \\ University of Connecticut, Storrs, Connecticut 06268
}

\begin{abstract}
In three experiments using the short-term memory distractor paradigm, Ss attempted to remember which three or four phalanges of the left hand had been stimulated and in what order. The experiments showed that forgetting increased as a function of trials, that such proactive effects could be eliminated by separating the successive trials by several minutes, that both verbal and nonverbal distractor tasks impaired retention, and that forgetting reached a maximum in approximately 6 sec. All of these results concur with those generally obtained for the short-term retention of verbal material. In addition, it was shown that the tactile recall was significantly poorer after an arithmetic distractor task presented visually than after the same task presented aurally. This result suggests an overlap between the mechanisms of tactile retention and the mechanisms of vision.
\end{abstract}

Most of what is known about the short-term memory (STM) performance of humans is based on experiments with verbal material, and it is often argued that STM is supported primarily by an acoustic-articulatory, or at least linguistic, code (e.g., Neisser, 1967). There is evidence, however, that the short-term retention function for verbal material is affected by the input modality (e.g., Kroll, Parkinson, \& Parks, 1972). Moreover, it can be demonstrated that the short-term forgetting of verbal material is more severe if the rehearsal-preventing task uses the modality in which the memory material was perceived (e.g., Salzberg, Parks, Kroll, \& Parkinson, 1971). Apparently an acoustic-articulatory code is not the only code [although it is unquestionably the most important code (Conrad, 1972)] that supports the short-term retention of verbal items; nonverbal codes can also be involved.

It is of some importance to determine the characteristics of short-term nonverbal codes and their relation to the verbal codes which have played the central role in theories of memory dynamics (e.g., Atkinson \& Shiffrin, 1968). As noted above, varying the modality in which verbal material is presented affords one means for examining nonverbal STM codes. But such a procedure must suffer because, where verbal material is involved, the verbal STM code is likely to dominate and, therefore, likely to occlude some of the characteristics of the nonverbal representation. What is needed are STM tasks which use nonverbal stimuli and thereby prohibit or at least reduce the usage of verbal coding and which, for purposes of comparison, are analogous to the STM tasks used to determine the properties of verbal retention.

Recent tactile analogues to verbal STM experiments (Gilson \& Baddeley, 1969; Schurman, Bernstein, \& Proctor, 1973; Sullivan \& Turvey, 1972) have pointed to certain differences between the retention of verbal and

*This report is based upon a thesis submitted for the MA degree by the first author under the supervision of the second author.

†Also, Haskins Laboratories, New Haven, Connecticut. Requests for reprints should be addressed to M.T. Turvey, Department of Psychology, U-20, University of Connecticut, Storrs, Connecticut 06268 . tactile material over brief periods. First, although proactive effects are strongly evident in verbal STM tasks (Wickens, 1970), Sullivan and Turvey (1972) did not observe proactive effects in the retention of single tactile stimuli in the STM distractor paradigm (Peterson \& Peterson, 1959). Second, in verbal STM experiments using the distractor paradigm, recall is perfect in the absence of a distractor, but in the tactile analogue unfilled retention periods still yield forgetting. At the very least, the forgetting of tactile stimulation over unfilled retention periods suggests a memory-sustaining capability for tactile stimulation different from that available to verbal material.

The present series of experiments investigated several aspects of short-term tactile memory. The first experiment looked for the operation of proactive interference (PI) in a tactile memory task in which the spatial location and the temporal order of four tactile stimuli had to be retained. The first experiment also examined the effect of various retention-interval tasks on tactile retention. The second experiment questioned whether the retention interval which characterizes verbal STM (cf. Peterson, 1966) similarly characterizes tactile STM. The third experiment examined the notion that at some level tactile retention involves the visual system (cf. Attneave \& Benson, 1969).

\section{GENERAL METHOD}

The general approach was based, in part, on a series of experiments by Bliss and colleagues (e.g., Bliss, Crane, Mansfield, $\&$ Townsend, 1966). Stimulation was given to the underside of the 12 phalanges of the left hand (excluding the thumbs). Each was numbered. The index finger phalanges, labeled in ink, were " 1 ," " 2 ," and " 3 ," starting with the farthest from the palm of the hand to the most proximal. The remaining phalanges were labeled similarly, using the remaining numbers through to " 12 ."

The $\mathbf{E}$ delivered either three or four stimuli per trial to approximately the center of the phalanges by means of a von Frey Hair 8 inserted into the end of a 1 -in. cork. A von Frey hair is a nylon filament used as a pressure controlling device. Following stimulation, E placed the von Frey hair into a 4-in. plastic ring on the table in S's view so that, when the recall cue was presented, the von Frey hair was readily available to S's right hand. Recall consisted of $S$ attempting to stimulate, using the 
von Frey hair, the phalanges which $\mathbf{E}$ had stimulated at the outset of the trial. The Ss were instructed to move the pointer during the recall period until they felt confident about the accuracy of their reports (see Sullivan \& Turvey, 1972).

Each $S$ sat to the right of $E$ and of a table upon which $S$ rested her left hand with the palm facing upward so that the 12 numbered phalanges were in clear view of $E$. In all three experiments, right-handed females served as Ss to minimize possible confounding resulting from handedness, laterality, or sex factors (Weinstein \& Sersen, 1961); E, also a female, ran each $S$ individually. During stimulus presentation, stimulus retention, and recall, Ss were not allowed to look at their hands.

The Ss were exposed to at least one, but to not more than three, practice trials, depending upon their individual needs to practice. When the Ss received four stimuli per trial, the recall scores were based on an eight-point scale, whereas a six-point scale provided the base when the Ss received three stimuli per trial. In either case, for each stimulus scored one point was awarded for the recall of the correct spatial locale and one point for the recall of its proper temporal order. A Kodak Carousel slide projector controlled by a tape timer provided procedural instructions and experimental timing for the Ss, with the exception of two conditions in Experiment III which used a Norelco cassette tape recorder for instruction delivery.

For all three experiments, Ss were female experimentally naive students from introductory psychology courses at the University of Connecticut who participated in the experiment in partial fulfillment of a course requirement.

\section{EXPERIMENT I}

While it is the case that verbal STM is sensitive to proactive effects, the evidence available so far suggests, as noted previously, that tactile STM is impervious to the influences of prior stimulation (e.g., Sullivan \& Turvey, 1972). However, the absence of significant proactive effects in previous tactile STM experiments may have been due, in part, to the use of only a single point of stimulation for retention per trial. Indeed, detectable proactive effects would not be expected in verbal STM experiments where the memory material for each trial consisted of only one consonant (cf. Melton, 1963). Since PI is clearly evident in verbal STM when several items are presented for retention on each trial (e.g., Wickens, 1970), it was hypothesized that tactile STM would be similarly sensitive to proactive effects when the retention load per trial was several points rather than one point of stimulation. In addition, it was hypothesized that, with several tactile stimuli to retain per trial, the activities performed by $S$ during the retention period would exert a significant influence on recall accuracy. The evidence of prior research is equivocal concerning the effects of competing retention-interval activity on tactile STM. The retention-interval activity used in the previous experiments has been the arithmetic task generally employed in verbal STM experiments (cf. Peterson \& Peterson, 1959). However, tactile retention is fundamentally nonverbal. Therefore, we might suppose that nonverbal retention-interval activities, engaging processes similar to those involved in tactile retention, would prove to be more damaging than verbal activities (cf. Brooks, 1967, 1968). In any event, the present experiment looked at the effects of both verbal and nonverbal retention-interval activities on tactile STM.

\section{Method}

Subjects. Forty Ss were assigned, by order of appearance at the laboratory, to one of four conditions, with $10 \mathrm{Ss}$ per condition.

Conditions. The four conditions were defined by four retention-interval tasks: rest, arithmetic task, tactile maze task, and visual maze task. For the rest condition Ss were informed that they should make an attempt during the retention interval to rehearse the points of stimulation "in their minds." In the arithmetic task condition, Ss were shown a series of seven two-digit numbers, each exposed for $2 \mathrm{sec}$. Upon seeing a number, Ss were instructed to say it aloud, to add the two components of the number together, to tell $E$ the answer, and to state whether the sum was odd or even. In the tactile maze condition, Ss were given a random-choice tactile maze. Without viewing the maze, Ss had to use the index finger of the right hand, i.e., the hand contralateral to that used for the memory task, to find their way from "start" to "finish." Upon hitting a blind alley, they had to retrace their paths until they found a correct path. At each new trial, Ss were required to begin the maze at "start." They were told that $\mathrm{E}$ would inform them if they reached "finish." At the beginning of the experiment, each $S$ was allowed to glance at the maze merely to understand its general nature.

In the visual maze condition, $S s$ were presented an $18 \times 12 \times 1 / 4$ in. peg board covering attached to a piece of styrofoam $18 \times 12 \times 1$ in. The visual maze was constructed so that its decision points were the same as those for the tactile maze. In this case, however, Ss were allowed to view the peg board side of the maze while they inserted with the right hand a hooked peg into successive holes, tracing right-angle paths to get from "start" to one of the holes at the top of the peg board. Diagonal paths were not allowed. In the styrofoam backing of the peg board were holes about $1 / 2$ in. deep designating the correct path. Therefore, if $S$ selected a correct peg hole, the peg would enter the hole freely, while, if S's choice were wrong, the peg would be stopped abruptly. As in the tactile maze condition, Ss were required to begin the maze at "start" at each new trial.

Procedure. Each S received five STM trials of the Brown-Peterson variety, with the retention-interval activity held constant from trial to trial. On each trial four successive tactile stimulations were given. A particular phalanx could not be used more than twice for any given $S$ or any given trial, and the same phalanx could not be used on successive trials. The following phalanges, chosen randomly, were used twice for each $S: 2,4,6$, $8,9,10,11$, and 12 . The quasirandomized stimuli sets were counterbalanced across trials to avoid possible order effects. Five trials were considered sufficient to produce PI if it did exist; in verbal STM experiments, the significant recall decrement occurs over the first three trials (e.g., Wickens, 1970).

The Ss were asked to keep their eyes fixed on the slide projection area where their procedural instructions were shown. They were informed that they would receive a series of trials, all following the same procedure. The Ss were not told the number of trials. The events comprising a trial were as follows. The word "READY," typed in block letters in the center of the first slide, appeared for $3 \mathrm{sec}$. A slide of red film paper followed for $4 \mathrm{sec}$, during which time E stimulated four of S's phalanges with the von Frey hair; $S$ was not allowed to observe E's activities. For the next $14 \mathrm{sec}, S$ engaged in one of the four experimental conditions. [In an earlier study, Sullivan and Turvey (1972) found that forgetting was maximal by a retention interval of $5 \mathrm{sec}$; therefore, an interval of $14 \mathrm{sec}$ was used to insure forgetting within any given trial.] In the rest condition a series of seven 2 -sec blank slides were projected. In the arithmetic task condition, seven 2 -sec slides of two-digit numbers occurred. For the conditions using the mazes, a 2 -sec slide stating "START 
MAZE" was followed by six 2-sec blank slides. These procedures stabilized conditions across Ss. The cue to recall was a $12-\mathrm{sec}$ slide of the words "RECALL STIMULI." The end of the recall period was designated by a 5 -sec blank slide which also served as the intertrial interval. An intertrial interval was used to insure the proper recording of S's recalls by $E$ and E's preparation for the ensuing trial. According to verbal PI studies (e.g., Kincaid \& Wickens, 1970), the intertrial interval between successive trials should be brief, since PI effects vary inversely with the intertrial interval. Within the 12-sec recall period, Ss were asked to try to recall the points of stimulation in the order in which they were delivered and to do this with their right hand by means of the pointer (i.e., the von Frey hair) provided by E.

\section{Results and Discussion}

Recall on each trial was scored in the manner described above. An analysis of variance revealed that the effect of trials was significant $[F(4,144)=4.22$, $\mathrm{p}<.01]$; performance on later trials tended to be poorer than performance on earlier trials, and this decline in performance across trials defines PI. The effect of the retention-interval task was also significant $[\mathrm{F}(3,160)=15.92, \mathrm{p}<.001]$, indicating that recall accuracy was dependent on what $\mathrm{S}$ did during the retention period. The analysis further revealed that there was no significant interaction between the two main effects $(F<1)$. The functions relating retention-interval activity to trials are presented in Fig. 1. It should be noted that scoring these data for spatial location alone revealed parallel retention percentages observed when accounting for both spatial location and temporal order: $\mathrm{R}=70 \%$ for the former and $64 \%$ for the latter, $\mathrm{AT}=$ $48 \%$ and $38 \%$, TMT $=44 \%$ and $34 \%$, and $\mathrm{VMT}=40 \%$ and $30 \%$.

Experiment I suggests, therefore, that tactile STM, like its verbal counterpart, can be said to be sensitive to PI. It was argued before that proactive effects might be observed in tactile STM under conditions where several tactile locations had to be retained per trial. The outcome of Experiment I would appear to confirm this argument. However, an alternative view of the proactive effects of the present experiment is that they arose not because tactile STM is sensitive to PI but because Ss coded and stored the tactile stimuli verbally. Fortunately, there are several reasons to believe that verbal coding did not play a significant role in the present experiment. First, Ss, on questioning, infrequently reported the use of a verbal strategy for remembering the tactile stimuli. Indeed, verbal coding in the present tactile task is both difficult and time consuming, particularly given the rapid rate of stimulation of one phalanx per second. (Readers are invited to try the task for themselves.) Second, if Ss were retaining the tactile input by verbal rehearsal, then concurrent performance of the maze tasks should have disturbed retention less than concurrent performance of the arithmetic task. The former would have afforded more opportunity for repeating verbal descriptions than the latter. If anything, the data suggest (see Fig. 1) that the maze tasks impaired retention more than the arithmetic task.

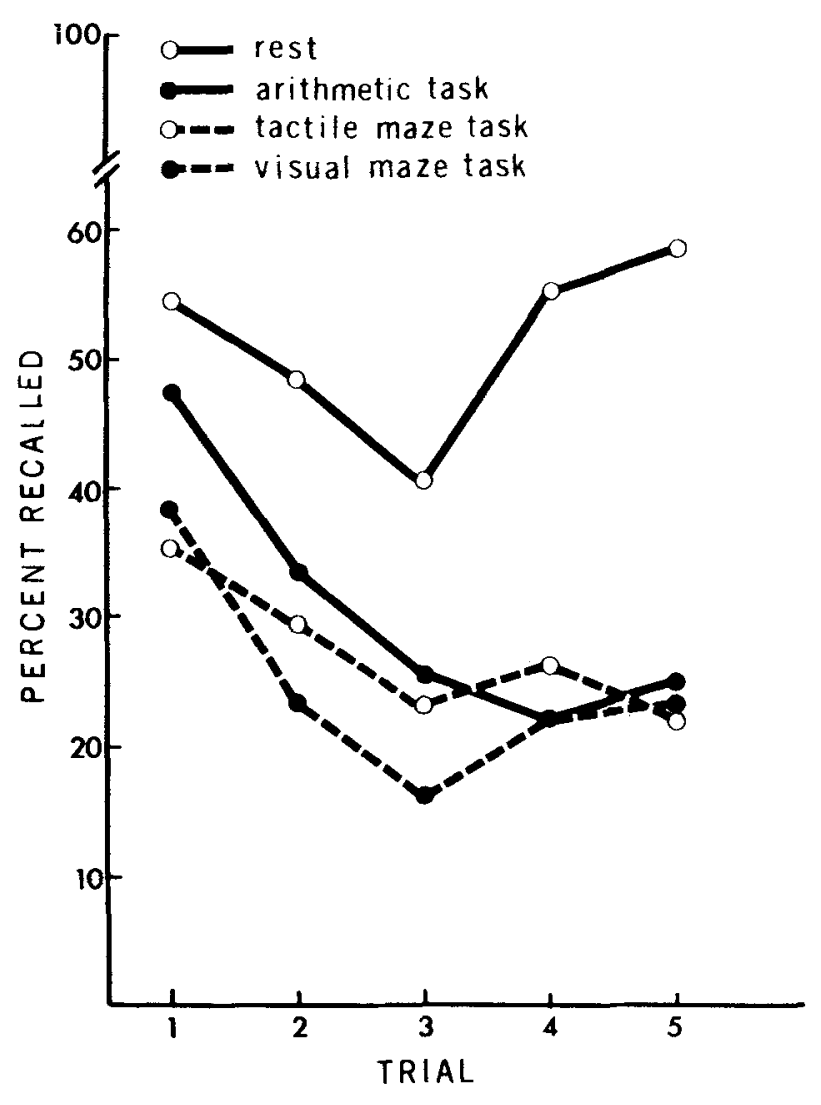

Fig. 1.

While the present experiment suggests that PI occurs in tactile STM, it also suggests that PI is not essential for tactile forgetting, since considerable forgetting occurred on the first trial of the series for all four conditions. Admittedly, the first trial was preceded by some practice of the tactile memory task, and this may have provided a source of interference. However, the time elapsing between practice and presentation of Trial 1 was on the order of several minutes, which should have been sufficient time for PI to dissipate. Significant forgetting on the first trial of a series is similarly found in verbal STM experiments and, therefore, there is reason to argue that neither tactile nor verbal forgetting over brief periods need be precipitated by proactive effects ( $\mathrm{cf}$. Baddeley \& Scott, 1971).

It is instructive to contrast the low level of recall in the rest condition of the present experiment with that which would be obtained if $S$ was asked to retain four successive speech events, say consonants, over an unfilled interval. Performance on the latter task would be, of course, virtually perfect. The implication is that STM processes for ordered, discrete tactile locations are not especially efficient, at least not as efficient as those available to linguistic material. The source of this inefficiency could be in any one, two, or three of the stages of initial coding, rehearsal, and recall.

The significant effect of the interpolated tasks in the present experiment suggests that rehearsal was important 
to the retention of four successive tactile stimuli. Of the three prior experiments on tactile STM, only one, that of Gilson and Baddeley (1969), found evidence for rehearsal in tactile STM, and that evidence was restricted, paradoxically, to the later retention periods. The positive finding of the present experiment was probably due to the use of several stimuli for retention per trial. The previous experiments (Gilson \& Baddeley, 1969; Schurman et al, 1973; Sullivan \& Turvey, 1972) had used a retention load of only one tactile location.

It was argued previously that the tactile stimuli of the present experiment were probably not verbally coded. Therefore, "rehearsal" defined as inner speech would be inappropriate. Following Posner (1966), a more useful view of rehearsal is that it refers to a class of (unidentified) processes which sustain memory over brief periods and which consume a portion of S's limited central processing capacity. The operations underlying the performance of the interpolated tasks would similarly require central processing capacity. Thus, in the present experiment the interpolated task, whether verbal or nonverbal, and the memory-sustaining operations on any one trial competed for S's limited attention; consequently, recall in the interpolated task conditions was notably poorer than in the rest condition. With respect to this discussion, it is important to note that the smaller the memory load the less the demand on central processing capacity (Shulman \& Greenberg, 1971).

\section{EXPERIMENT II}

The second experiment was designed to examine the short-term forgetting function for serial tactile stimuli. In addition, the experiment sought to determine whether the significant trials effect observed in Experiment $I$ could be eliminated if the interval between the trials was lengthened. In verbal STM experiments, spacing the trials reduces PI (Kincaid \& Wickens, 1970).

\section{Method}

Subjects. Twenty-four Ss were allocated, in order of appearance at the laboratory, to one of three conditions, with eight Ss to each condition.

Procedure. The procedure followed that of Experiment I with the following exceptions. First, three phalanges, rather than four, were stimulated per trial within a $3-\mathrm{sec}$ period. Bliss et al (1966) have shown that the immediate memory span in the present task is approximately 4.5 locations. By reducing the memory load to well within the memory span, it was hoped that a higher level of performance than that of Experiment I would be obtained. Second, only one interpolated task, the arithmetic task, was used for all three conditions. This task was chosen because it was the most easily controlled of the three tasks used in Experiment I. Third, each $S$ received four rather than five trials.

Four three-stimuli sequences were used, with the constraint for each sequence that no phalanx could be repeated and horizontally or vertically adjacent phalanges could not be stimulated. The four sequences were balanced across $S$ s within a condition so that each sequence occurred twice in each trial position. A Carousel projector timed the events and the interval between the end of one trial, i.e., the end of the recall period and the beginning of the next was set at $2 \mathrm{~min}$. Within the four-trial series, each $S$ received only one retention interval. The retention intervals used were 0,6 , and $28 \mathrm{sec}$, and these defined the three conditions of the experiment.

\section{Results and Discussion}

An analysis was initially conducted on the effect of trials. Averaged across Ss and across conditions, the recall accuracy on the four successive trials was $53 \%$, $47 \%, 48 \%$, and $47 \%$. The analysis proved insignificant, suggesting that PI effects had been eliminated by the procedure of spacing the trials. The recall data were then averaged across trials within a condition and a simple analysis of variance revealed that the main effect of retention interval was significant $[F(2,21)=9.53$, $\mathrm{p}<.005]$. Recall in the $0-, 6-$, and 28 -sec conditions was, respectively, $67 \%, 41 \%$, and $38 \%$. When these data were scored for position only, recall in the 0-, 6-, and 28 -sec conditions was, respectively, $80 \%, 62 \%$, and $57 \%$.

A typical interpretation of STM functions of the kind obtained in the present experiment is that they reflect two memory processes: a short-term component whose contribution to memory performance declines rapidly over the first few seconds and a relatively longer term component whose contribution to performance is virtually invariant over brief retention periods (cf. Peterson, 1966). What is particularly important about the forgetting function obtained in the present experiment is that it compares favorably, both in its general form and in its point of maximal forgetting, with the forgetting functions obtained with verbal material in the distractor paradigm (Baddeley \& Scott, 1971). The importance of this observation lies in its implication that common principles underlie the forgetting of verbal and nonverbal stimulation under conditions of filled retention periods. It should be noted that Sullivan and Turvey (1972) and Schurman et al (1973) reported functions for tactile retention of single stimuli very similar to those reported here. In those experiments tactile forgetting reached an asymptote within $5 \mathrm{sec}$.

\section{EXPERIMENT III}

The point of departure for the third experiment was the notion that a visual-spatial code might underlie the retention of tactile location (Attneave \& Benson, 1969). On this notion it was hypothesized that tactile STM would be more affected by an interpolated task presented visually than by the same task presented aurally. This hypothesis derives from experiments showing that visual activity is more antagonistic than aural activity to the construction and retention of spatial representations. Thus, Brooks (1967) showed that the spatial coding of a message was poorer when the message was read than when it was heard. More recently, den Hyer and Barrett (1971) reported that the short-term retention of the location of letters in a visually presented matrix was selectively affected by a 
visual interpolated task, whereas the identity of the letters was selectively affected by an auditory task.

The third experiment was similar in its design to the two preceding experiments: Ss were required to retain a set of ordered tactile locations while performing an arithmetic task. The important difference was that the arithmetic task was presented either aurally, as before, or visually. The expected outcome was that tactile recall would be poorer in the latter case.

\section{Method}

Subjects. Each of 32 Ss was allocated to one of four conditions by order of appearance at the laboratory, with $8 \mathrm{Ss}$ per condition. No $S$ had participated in the previous experiments.

Procedure. The four conditions were auditory arithmetic task (AAT), visual arithmetic task (VAT), auditory rest (AR), and visual rest (VR). During the retention interval of the AAT condition, $S$ heard a series of eight two-digit numbers, each presented for $2 \mathrm{sec}$. In the VAT condition the same digit pairs were presented visually at the same rate. During the AR retention period, $S$ heard a series of eight clicks at 2 -sec intervals; during the VR retention period, $S$ saw a series of eight blank slides, each of 2-sec duration. For both auditory conditions, the presentation of the three tactile stimuli per trial coincided with a steady state tone; in the visual conditions the tactile stimulation coincided with a red slide. No $S$ was allowed to look at her left hand during the experiment. In addition, $S s$ in the auditory conditions kept their eyes closed during the retention interval in order to eliminate possible confounding visual effects.

Each of the four conditions used a 2-sec ready signal, a 3-sec tactile stimulation period, a 16-sec retention interval, a 12-sec recall period, and a 2 -min intertrial interval. In each condition, each $S$ was given eight trials, thus yielding 64 observations per condition. On completion of the experiment, each $S$ was questioned about the strategy she used to retain the tactile stimuli.

\section{Results and Discussion}

A representation of the recall performance in each condition is provided by Fig. 2. An analysis of variance showed that there was a significant difference between performing the arithmetic task and resting $[F(1,28)=33.45, p<.001]$. More important, the auditory/visual difference and the interaction between modality and task proved significant $[\mathrm{F}(1,28)=5.07$, $\mathrm{p}<.05$ and $\mathrm{F}(1,28)=3.35, \mathrm{p}<.025$, respectively] . A Duncan's multiple range test revealed that, at the $p=.05$ level, $\mathrm{VR}=\mathrm{AR}>\mathrm{AAT}>\mathrm{VAT}$. A comparison of recall levels when these data were scored for correct spatial location independent of correct temporal order and when they were scored for correct location and order yielded the following: VR $=76 \%$ for the former and $75 \%$ for the latter, $\mathrm{AR}=76 \%$ and $75 \%$, VAT $=48 \%$ and $41 \%$, and $\mathrm{AAT}=68 \%$ and $61 \%$. This suggests that the forgetting effects observed in the present experiment were due more to the loss of spatial location than to the loss of serial order.

The conclusion we would like to draw from this experiment is that tactile retention is impaired more by concurrent visual activity than by concurrent aural activity and, therefore, can be said to share processes more in common with vision than with audition.

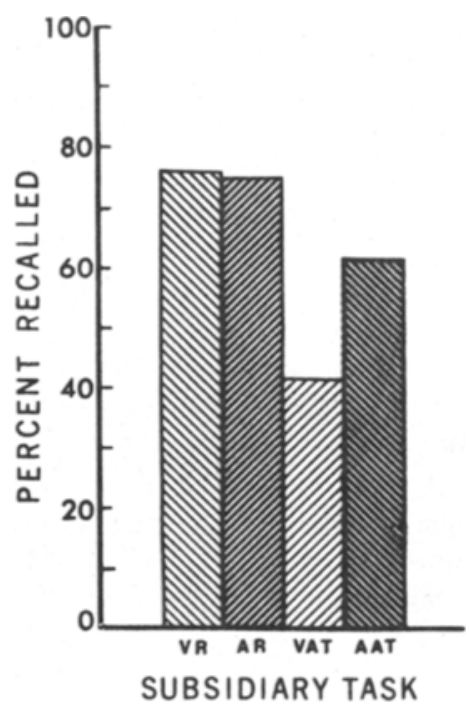

Fig. 2.

However, quite to the contrary, one could conclude that the difference between the VAT and AAT conditions was due to a difference in the difficulty of the two interpolated tasks rather than to a difference in the modalities of their presentation. In short, the arithmetic task presented visually entailed operations over and above those of that task presented aurally, and these extra operations resulted in greater demands on S's limited processing capacity. This argument, however, is partially offset by the fact that performance on the arithmetic task was virtually identical for the two conditions. Performance on the digit-pair addition and classification task was scored as follows: one point for correctly repeating the two digits, one point for correctly adding them, and one point for reporting correctly the odd/even classification. On this measure the overall accuracy of performance in the VAT and AAT conditions was $90 \%$ and $94 \%$, respectively. Of course, it could still be argued that, while performance was equivalent in the two conditions, the effort needed to achieve that level of performance in the VAT condition was greater than that needed in the AAT condition.

At best, the present data may be taken as only suggestive of a relation between the brief retention of spatially arranged tactile stimulation and visual processes. But data from other sources point in a similar direction: Brooks (1968, Experiment VII) showed that visualizing the spatial characteristics of a previously memorized diagram and signaling those characteristics by means of tactually monitored movements are, apparently, conflicting operations; Attneave and Benson (1968) demonstrated that learning responses to tactile stimuli delivered in fixed locations is better with unrestricted than with restricted vision.

On completion of the present experiment, Ss were asked to report their strategies used to retain the tactile 
arrays. On the basis of their reports, the Ss were divided into two groups: those who reported never using any form of a verbal code on any of the trials and those who reported using some form of a verbal code on some of the trials. There were $16 \mathrm{Ss}$ in the nonverbal group and $15 \mathrm{Ss}$ in the verbal group (E forgot to collect a report from one $S$ ). The average STM performance was equivalent for the two groups: nonverbal, $61 \%$; verbal, $63 \%$.

Verbal strategies consisted of trying to name either the finger stimulated or the part of the finger stimulated, i.e., top, middle, or bottom. In general, Ss reported that they found their verbal coding difficult and, for the most part, inadequate. The nonverbal strategies involved either attempted to remember the "afterimage" of the tactile stimulation or attempts to image the spatial arrangement. There were also five Ss in the nonverbal group who were not aware of using any strategy whatsoever. In the VAT condition, there were three verbal coders and five nonverbal coders; in the AAT condition, there were four verbal coders and four nonverbal coders. Thus, the significant difference between the two conditions was probably not due to differences in coding strategies.

By way of summary, the present series of experiments has shown that STM for serial tactile stimuli is sensitive to the effects of PI, that forgetting of serial tactile stimuli over brief periods is characterized by a rapid decline to asymptote within $6 \mathrm{sec}$, and that the retention of tactile stimulation might be dependent on mechanisms closely related to vision.

\section{REFERENCES}

Atkinson, R. C., \& Shiffrin, R. M. Human memory: A proposed system and its control processes. In K. W. Spence and J. T Spence (Eds.), Psychology of learning and motivation: Advances in research and theory. Vol. 2. New York: Academ ic Press, 1968

Attneave, F., \& Benson, B. Spatial coding of tactual stimulation. Journal of Experimental Psychology, 1969, 81, 216-222.

Baddeley, A. D., J \& Scott, D. Short-term forgetting in the absence of proactive interference. Quarterly Journal of Psy chology, 1971, 23, 275-283.
Bliss, J. C., Crane, H. D., Mansfield, P. K., \& Townsend, J. T. Information available in brief tactile presentation. Perception \& Psychophy sics, 1966, 1, 273-283.

Brooks, $L$. $R$. The suppression of visualization by reading. Quarterly Journal of Experimental Psychology, 1967, 19, 289-299.

Brooks, L. R. Spatial and verbal components of the act of recall. Canadian Journal of Psy chology, 1968, 22, 349-368.

Conrad, R. Speech and reading. In J. F. Kavanagh and I. G. Mattingly (Eds.), Language by ear and by eye. Boston: MIT Press, 1972 .

den Heyer. K.. \& Garrett, B. Selective loss of visual and verbal information in STM by means of visual and verbal interpolated tasks. Psychonomic Science, 1971, 25, 100-102.

Gilson, E. Q., \& Baddeley, A. D. Tactile short-term memory, Quarterly Journal of Experimental Psychology, 1969,21, 180-184.

Kincaid, J. P., \& Wickens, D. D. Temporal gradient of release from proactive inhibition. Journal of Experimental Psychology, 1970, 86, 313-316.

Kroll, N. E. A., Parkinson, S. R., \& Parks, T. E. Sensory and active storage of compound visual and auditory stimuli. Journal of Experimental Psychology, 1972, 95, 32-38.

Melton, A. W. Implications of short-term memory for a general theory of memory. Journal of Verbal Learning \& Verbal Behavior, 1963, 2, 1-21.

Neisser, U, Cognitive psychology. New York: Appleton-Century-Crofts, 1967.

Peterson, L. R. Short-term verbal memory and learning. Psychological Review, 1966, 73, 193-207.

Peterson, L, R. \& Peterson, M. J. Short-term retention of individual verbal items. Journal of Experimental Psychology, $1959,58,193-198$.

Posner, M. I. Components of skilled performance. Science, 1966 $152,1712-1718$

Salzberg, P. N. Parks, T. E., Kroll, N. E. A., \& Parkinson, S. R. Retroactive effects of phonemic similarity on short-term recall of visual and auditory stimuli. Journal of Experimental Psy chology, 1971, 91, 43-46.

Schurman, D. L., Bernstein, I. H., \& Proctor, R. W. Modality-specific short-term storage for pressure. Bulletin of the Psychonomic Society, 1973, 1, 71-74.

Shulman, H. G., \& Greenberg, S. N. Perceptual deficit due to division of attention between memory and perception. Journal of Experimental Psychology, 1971, 88, 171-176.

Sullivan, E. V., \& Turvey, M. T. Short-term retention of tactile stimulation. Quarterly Journal of Experimental Psychology, $1972,24,253-261$.

Weinstein, S., \& Sersen, E. A. Tactual sensitivity as a function of handedness and laterality. Journal of Comparative \& Phy siological Psychology, 1961, 54, 665-669.

Wickens, D. D. Encoding categories of words: An empirical approach to meaning. Psychological Review, 1970, 77, 1-15.

(Received for publication November 28, 1973 revision received January 28,1974 .) 